CIEA-GR-9407

ICN-UNAM-9405

\title{
GEOMETRY OF DEFORMATIONS OF RELATIVISTIC MEMBRANES
}

\author{
Riccardo Capovilla ${ }^{(1)}$ and Jemal Guven ${ }^{(2)}$ \\ (Revised Version) \\ (1) Departamento de Física \\ Centro de Investigación y de Estudios Avanzados del I.P.N. \\ Apdo. Postal 14-740, 07000 México, D. F., MEXICO \\ (capo@fis.cinvestav.mx) \\ (2) Instituto de Ciencias Nucleares \\ Universidad Nacional Autónoma de México \\ Apdo. Postal 70-543, 04510 México, D. F., MEXICO \\ (guven@roxanne.nuclecu.unam.mx)
}

\begin{abstract}
A kinematical description of infinitesimal deformations of the worldsheet spanned in spacetime by a relativistic membrane is presented. This provides a framework for obtaining both the classical equations of motion and the equations describing infinitesimal deformations about solutions of these equations when the action describing the dynamics of this membrane is constructed using any local geometrical worldsheet scalars. As examples, we consider a Nambu membrane, and an action quadratic in the extrinsic curvature of the worldsheet.
\end{abstract}

PACS: $98.80 . \mathrm{Cq}, 13.70 .+\mathrm{k}, 98.80 \mathrm{Hw}$ 


\section{INTRODUCTION}

A useful approximate description of the dynamics of many physical systems is to model them as relativistic membranes of an appropriate dimension. The construction of the corresponding phenomenological action determining the dynamics of the membrane involves the selection of an appropriate linear combination of the geometrical scalars of its worldsheet. At lowest order, this action is proportional to the intrinsic volume of the worldsheet, and it has come to be known as the Nambu action. If the approximation stops here, the classical trajectory of the membrane will be an extremal surface of the background spacetime. A large body of information has accumulated on the dynamics of geometrically symmetrical extremal solutions (see e.g. Ref. [1], for a review in the context of cosmology). To place these solutions in proper context, however, their stability needs to be examined both with respect to classical and quantum mechanical perturbations propagating on What becomes clear when this is attempted for even the simplest models is that a manifestly covariant formalism to describe the evolution of these perturbations which is also independent of the particular symmetry of the membrane is desirable. This problem was approached for Nambu membranes by one of the authors in Ref.[3] and, independently, using similar techniques in Refs.[4] and [5].* The perturbation is described by a system of coupled linear wave equations, one for the projection of the infinitesimal deformation in the worldsheet onto each normal direction, which can be considered as scalar fields living on the worldsheet. In this way, a perturbative framework for examining the stability of any system described by the Nambu action is provided.

The analysis presented in Refs. [3,4] and [5] was tailored to describe extremal surfaces. For some time, however, it has been realized that extrinsic curvature additions or corrections to the Nambu action can have a dramatic influence on the dynamics on short length scales $[7,8]$. In particular, when such corrections are introduced the development of cusps or kinks on the membrane appears to be inhibited on these scales. These corrections may arise in a more realistic truncation of the underlying field theory, or may be induced by quantum mechanical fluctuations.

The equations of motion which correspond to a generic action which is quadratic in the extrinsic curvature, are typically hyperbolic equations which are fourth order in deriva-

* The relevant mathematical formalism was developed by mathematicians much earlier in the context of minimal surfaces [6]. 
tives of the embedding functions describing the worldsheet. To derive these equations and their subsequent linearizations, one could attempt to imitate the analysis applied earlier to extremal surfaces. However, by following this case by case approach, one can easily lose sight of the fact that there is a solid kinematical structure underpinning these equations which is entirely independent of the underlying dynamics. In this paper, we develop such a kinematical framework for describing deformations of an arbitrary worldsheet. Relevant earlier investigations in this direction are Refs.[5,8,9] and [10]. In Ref.[10] Hartley and Tucker exploit very elegant exterior differential techniques to derive the equations of motion for relativistic membranes. This language could, potentially, provide a very powerful geometrical approach to the description of deformations.

Our effort divides naturally into an examination of the deformation of the intrinsic and of the extrinsic geometry of the worldsheet. Once this is done, both the equations of motion, and the equations describing the dynamics of deformations about classical solutions can be constructed in Lego block fashion, by assembling the various kinematical ingredients.

The paper is organized as follows. To establish our notation, we begin in section II by summarizing the well known classical kinematical description provided by the GaussWeingarten equations of an embedded timelike worldsheet of dimension $D$ in a spacetime of dimension $N$, in terms of its intrinsic and extrinsic geometry $[11,12,13]$. There are two structures which describe the extrinsic geometry. One of these is given by the extrinsic curvatures, and is well understood. The other structure, which we call the extrinsic twist potential, only features when the co-dimension of the worldsheet is two or higher. The extrinsic twist plays a subtle role related to the covariance of the description of the geometry under rotations of the normals to the worldsheet.

In this same kinematical spirit, in sections III and IV we describe the deformation of the worldsheet. There are analogues of the Gauss-Weingarten equations which are useful for identifying the structures associated with such deformations. The description of the deformation divides naturally into two parts. The deformation of the intrinsic geometry is very simple to describe. Indeed, for example, the deformations of the worldsheet metric provide a geometrically satisfying definition of the extrinsic curvatures. The description of the deformation of the extrinsic geometry is less simple. One reason for this is because the naive deformation of the structures associated with the extrinsic geometry do not transform covariantly under normal frame rotations. By examining the deformation of the 
normal vectors (the analogue of the Weingarten equations) we can identify a 'connection' which guarantees the manifest covariance of the deformation of such structures under normal frame rotations. It turns out, however, that this connection does not appear in any physical quantity, and thus it does not need to be calculated.

In section $\mathrm{V}$, we apply this kinematical framework to some phenomenological actions of physical interest. We consider first the familiar Nambu action, to show how our analysis reproduces the results of Ref. [3] and [4]. Next, we consider an action quadratic in the extrinsic curvature. We derive the equations of motion, and the equations that describe the dynamics of deformations about classical solutions in the case the background spacetime is Minkowski space. We conclude in section VI with a brief discussion.

We confine our attention to closed membranes without physical boundaries.

\section{MATHEMATICS OF THE EMBEDDING OF THE WORLDSHEET}

In this section we provide an overview of the well known mathematical description

of the worldsheet of a membrane viewed as an embedded surface in a fixed background spacetime $[11,12,13]$.

Let us consider an oriented timelike worldsheet $m$ of dimension $D$ described by the embedding

$$
x^{\mu}=X^{\mu}\left(\xi^{a}\right)
$$

$\mu=0, \cdots, N-1$, and $a=0, \cdots, D-1$, in an $N$-dimensional spacetime $M$, endowed with the metric $g_{\mu \nu}$. The $D$ vectors

$$
e_{a}:=X_{, a}^{\mu} \partial_{\mu}
$$

form a basis of tangent vectors to $m$ at each point of $m$. The metric induced on the world sheet is then given by

$$
\gamma_{a b}=X_{, a}^{\mu} X_{, b}^{\nu} g_{\mu \nu}=g\left(e_{a}, e_{b}\right)
$$

The signature of $\gamma_{a b}$ is taken to be $\{-,+, \ldots,+\}$.

Let $n^{i}$ denote the $i^{\text {th }}$ unit normal to the worldsheet, $i=1, \cdots, N-D$, defined by 


$$
g\left(n^{i}, n^{j}\right)=\delta^{i j}, \quad g\left(e_{a}, n^{i}\right)=0
$$

It is important to emphasize that these equations define the normal vector fields $n^{i}$ only up to a $O(N-D)$ rotation, and up to a sign.

Normal vielbein indices are raised and lowered with $\delta^{i j}$ and $\delta_{i j}$, respectively, whereas tangential indices are raised and lowered with $\gamma^{a b}$ and $\gamma_{a b}$, respectively.

The collection of vectors $\left\{e_{a}, n^{i}\right\}$ can be used as a basis for the spacetime vectors appropriate for the geometry under consideration.

We define the worldsheet projections of the spacetime covariant derivatives with $D_{a}:=$ $e_{a}^{\mu} D_{\mu}$, where $D_{\mu}$ is the (torsionless) covariant derivative compatible with $g_{\mu \nu}$. Let us now consider the worldsheet gradients of the basis vectors $\left\{e_{a}, n^{i}\right\}, D_{a} e_{b}$ and $D_{a} n^{i}$. These spacetime vectors can always be decomposed with respect to the basis, ${ }^{*}$

$$
\begin{aligned}
& D_{a} e_{b}={\gamma_{a b}}^{c} e_{c}-K_{a b}^{i} n_{i}, \\
& D_{a} n^{i}=K_{a b}^{i} e^{b}+\omega_{a}^{i j} n_{j} .
\end{aligned}
$$

These kinematical expressions, generalizing the classical Gauss-Weingarten equations, describe completely the extrinsic geometry of the worldsheet.

The $\gamma_{a b}^{c}$ are the connection coefficients compatible with the worldsheet metric $\gamma_{a b}$,

$$
\gamma_{a b}{ }^{c}=g\left(D_{a} e_{b}, e^{c}\right)=\gamma_{b a}{ }^{c}
$$

The quantity $K_{a b}{ }^{i}$ is the $i^{\text {th }}$ extrinsic curvature of the worldsheet,

$$
K_{a b}^{i}=-g\left(D_{a} e_{b}, n^{i}\right)=K_{b a}^{i} .
$$

The symmetry in the tangential indices of these quantities is a consequence of the integrability of the tangential basis $\left\{e_{a}\right\}$.

The normal fundamental form, or extrinsic twist potential, of the worldsheet is defined by

* To avoid confusion, we adopt the notation $\omega$ (adopted by Maeda and Turok in Ref.[6]) instead of $T$ as was used in Ref.[3]) for the twist potential, and $\Omega$ (adopted by Carter in Ref.[5]) for the corresponding curvature. 


$$
\omega_{a}^{i j}=g\left(D_{a} n^{i}, n^{j}\right)=-\omega_{a}^{j i}
$$

In the familiar case of a hypersurface embedding, $D=N-1$, the extrinsic twist vanishes identically. The geometrical meaning of $\omega_{a}^{i j}$ can be understood by recalling that there is the freedom to perform local rotations of the normal frame, $\left\{n^{i}\right\}$. With respect to the rotation, $n^{i} \rightarrow O^{i}{ }_{j} n^{j}, \gamma_{a b}{ }^{c}$ transforms as a scalar, and $K_{a b}{ }^{i}$ transforms as a vector. The extrinsic twist potential, $\omega_{a}^{i j}$, transforms as a connection,

$$
\omega_{a} \rightarrow O \omega_{a} O^{-1}+O_{, a} O^{-1}
$$

As discussed in detail in Ref.[3], for example, it can be considered as the gauge field associated with the normal frame rotation group. It is desirable to implement this covariance in a manifest way. Let $\nabla_{a}$ be the (torsionless) covariant derivative compatible with $\gamma_{a b}$ induced on $m$. We introduce a new worldsheet covariant derivative $\tilde{\nabla}_{a}$ defined on fields transforming as tensors under normal frame rotations as follows,

$$
\tilde{\nabla}_{a} \Phi_{j}^{i}:=\nabla_{a} \Phi_{j}^{i}-\omega_{a}^{i k} \Phi_{k j}-\omega_{a j k} \Phi^{i k}
$$

We also introduce the curvature associated with $\omega_{a}^{i j}$ with

$$
\Omega_{a b}^{i j}:=\nabla_{b} \omega_{a}^{i j}-\nabla_{a} \omega_{b}^{i j}-\omega_{b}^{i k} \omega_{a k}^{j}+\omega_{a}^{i k} \omega_{b k}^{j}
$$

Note that when $D=1, \Omega_{a b}{ }^{i j}=0$ so that $\omega_{a}^{i j}$ is pure gauge, at least locally. We also know that when $D=N-1, \omega_{a}^{i j}=0$. When $D=N-2$, the gauge group is $O(2)$ with a single generator. We conclude that the non-abelian (or non-linear) character of $\omega_{a}^{i j}$ displayed in an arbitrary dimension does not manifest itself in spacetime dimensions lower than five.

Given some specification of intrinsic and extrinsic geometry Eqs.(2.5) will not generally be consistent with any embedding because $X^{\mu}(\xi)$ is over specified by these equations. Consistency will require that the intrinsic and extrinsic geometry satisfy the Gauss-Codazzi, Codazzi-Mainardi, and Ricci integrability conditions,

$$
\begin{gathered}
g\left(R\left(e_{b}, e_{a}\right) e_{c}, e_{d}\right)=\mathcal{R}_{a b c d}-K_{a c}{ }^{i} K_{b d i}+K_{a d}{ }^{i} K_{b c i} \\
g\left(R\left(e_{b}, e_{a}\right) e_{c}, n^{i}\right)=\tilde{\nabla}_{a} K_{b c}{ }^{i}-\tilde{\nabla}_{b} K_{a c}{ }^{i}
\end{gathered}
$$




$$
g\left(R\left(e_{b}, e_{a}\right) n^{i}, n^{j}\right)=\Omega_{a b}{ }^{i j}-K_{a c}{ }^{i} K_{b}{ }^{c j}+K_{b c}{ }^{i} K_{a}{ }^{c j} .
$$

We use the notation $g\left(R\left(Y_{1}, Y_{2}\right) Y_{3}, Y_{4}\right)=R_{\alpha \beta \mu \nu} Y_{1}^{\mu} Y_{2}^{\nu} Y_{3}^{\beta} Y_{4}^{\alpha} \cdot R_{\beta \mu \nu}^{\alpha}$ is the Riemann tensor of the spacetime covariant derivative $D_{\mu}$, whereas $\mathcal{R}^{a}{ }_{b c d}$ is the Riemann tensor of the worldsheet covariant derivative $\nabla_{a}$. Note that Eq.(2.12c) possesses no non-trivial contractions. In particular, it is vacuous when $D=1$, and when $D=N-1$.

These equations can be obtained directly from the Gauss-Weingarten equations, by taking a second spacetime covariant derivative projected onto the worldsheet, subtracting the same equation with the indices on the derivatives switched, and exploiting the spacetime Ricci identity.

In de Sitter spacetime,

$$
R_{\mu \nu \alpha \beta}=H^{2}\left(g_{\mu \alpha} g_{\nu \beta}-g_{\mu \beta} g_{\nu \alpha}\right)
$$

so that

$$
g\left(R\left(e_{b}, e_{a}\right) e_{c}, e_{d}\right)=H^{2}\left(\gamma_{a c} \gamma_{b d}-\gamma_{a d} \gamma_{b c}\right) .
$$

The right hand sides of Eqs.(2.12b) and (2.12c) both vanish.*

The appearance of the curvature of the extrinsic twist potential, $\Omega_{a b}{ }^{i j}$, in the Ricci integrability condition, Eq.(2.12c), provides us with additional information about the extrinsic twist itself. First, note that, for a given spacetime, Eq. (2.12c) implies that the curvature $\Omega_{a b}{ }^{i j}$ is completely determined once the intrinsic geometry and the extrinsic curvatures are specified. In fact, as was emphasized by Carter, $\Omega_{a b}{ }^{i j}$ is the conformally invariant tracefree part of the squared extrinsic curvature [13,14]. This equation also provides the necessary and sufficient conditions that the extrinsic twist can be gauged away,

$$
g\left(R\left(e_{a}, e_{b}\right) n^{i}, n^{j}\right)-K_{a c}{ }^{i} K_{b}{ }^{c j}+K_{b c}{ }^{i} K_{a}{ }^{c j}=0 .
$$

* From Eq. (2.12a), it is clear that the necessary and sufficient condition that the worldsheet will also be a de Sitter space is that

$$
K_{a c}{ }^{i} K_{b d i}-K_{a d}{ }^{i} K_{b c i} \propto\left(\gamma_{a c} \gamma_{b d}-\gamma_{a d} \gamma_{b c}\right) .
$$


In particular, in de Sitter spacetime, if all but one $K_{a b}^{i}$ vanish, then the antisymmetric product of extrinsic curvature tensors vanishes, and the integrability condition is satisfied automatically.

\section{DEFORMATIONS OF THE INTRINSIC GEOMETRY}

In the previous section, we have described the characterization of a single embedded surface in spacetime, in terms of its intrinsic and extrinsic geometry.

Let us now consider the neighboring surface described by a deformation of $m$,

$$
x^{\mu}=X^{\mu}\left(\xi^{a}\right)+\delta X^{\mu}\left(\xi^{a}\right) .
$$

We can decompose the infinitesimal deformation vector field $\delta X^{\mu}$ with respect to the spacetime basis $\left\{e_{a}, n^{i}\right\}$, as,

$$
\delta X=\Phi^{a} e_{a}+\Phi^{i} n_{i} .
$$

The tangential projection can always be identified with the action of a worldsheet diffeomorphism, $\delta X^{\mu}=\Phi^{a} X_{a}^{\mu}$, and so will subsequently be ignored. The physically observable measure of the deformation is therefore provided by the projection of $\delta X^{\mu}$ orthogonal to $m$, characterized by the $N-D$ scalar fields $\Phi^{i}$.

Our task will be to express the deformation of the geometrical structures introduced in section II as linear combinations of the scalar fields $\Phi^{i}$, and their covariant derivatives, $\tilde{\nabla}_{a} \Phi^{i}, \tilde{\nabla}_{a} \tilde{\nabla}_{b} \Phi^{i}, \ldots$ We make use of the covariant derivative defined in (2.10), because $\Phi^{i}$ transforms as a vector under normal frame rotations.

In this section we consider the deformation of the intrinsic geometry of the worldsheet under a deformation in the embedding. The displacement $\delta X^{\mu}$ in the embedding induces a displacement in the tangent basis $\left\{e_{a}\right\}$. In light of the discussion above, let $\delta=\Phi^{i} n_{i}$, and consider the gradients of $\left\{e_{a}\right\}$ along the vector field $\delta$, defined with $D_{\delta}:=\delta^{\mu} D_{\mu}$. We can always expand $D_{\delta} e_{a}$ with respect to the spacetime basis $\left\{e_{a}, n_{i}\right\}$, in a way analogous to the Gauss equation, (2.4a), as follows,

$$
D_{\delta} e_{a}=\beta_{a b} e^{b}+J_{a j} n^{j} .
$$

Comparison with the Gauss equation shows that the quantity $\beta_{a b}$, defined by 


$$
\beta_{a b}=g\left(D_{\delta} e_{a}, e_{b}\right)=\beta_{b a},
$$

appears in the same position as $\gamma_{a b}{ }^{c}$. The quantities $J_{a i}$ are defined by

$$
J_{a i}=g\left(D_{\delta} e_{a}, n_{i}\right),
$$

and appear in the same position as $K_{a b}{ }^{i}$ in the Gauss equation. We note that $\beta_{a b}$ transforms as a scalar under normal frame rotations, whereas $J_{a i}$ transforms as a vector.

In order to express $\beta_{a b}$ and $J_{a i}$ in terms of $\Phi^{i}$ and its covariant derivatives it is crucial to recognize that, for all infinitesimal deformations of the worldsheet [15], one has

$$
D_{\delta} e_{a}=D_{a} \delta .
$$

In words, this equation follows from the equality of the gradient along the deformation vector field $\delta$ of the tangential basis $\left\{e^{a}\right\}$, with the change of $\left\{e^{a}\right\}$ induced by the displacement of the worldsheet.

Using Eq. (3.5), it is easy to show that,

$$
\begin{gathered}
\beta_{a b}=g\left(D_{\delta} e_{a}, e_{b}\right)=g\left(D_{a} \delta, e_{b}\right)=g\left(D_{a} n^{i}, e_{b}\right) \Phi_{i}=K_{a b}{ }^{i} \Phi_{i}, \\
J_{a i}=g\left(D_{\delta} e_{a}, n_{i}\right)=g\left(D_{a} \delta, n_{i}\right)=g\left(D_{a} n^{j}, n^{i}\right) \Phi_{j}+\nabla_{a} \Phi_{i}=\tilde{\nabla}_{a} \Phi_{i} .
\end{gathered}
$$

Therefore, the gradients along the deformation of the tangential vectors depend on the values of the scalar fields $\Phi^{i}$, and on their first derivatives along the worldsheet.

The deformation in the induced metric on $m$ is just twice $\beta_{a b}$,

$$
D_{\delta} \gamma_{a b}=D_{\delta} g\left(e_{a}, e_{b}\right)=2 g\left(e_{a}, D_{\delta} e_{b}\right)=2 \beta_{a b}=2 K_{a b}{ }^{i} \Phi_{i} .
$$

In fact, this equation encodes the geometrical role of $K_{a b}^{i}$. It is half the change induced in the worldsheet metric per unit proper deformation of the worldsheet along the $i^{\text {th }}$ normal direction.

This is all we need to know about the deformation of the intrinsic geometry, if we are only interested in the deformation of extremal surfaces. However, one might also be interested in more general theories that contain scalars constructed with the worldsheet curvature tensor, $\mathcal{R}^{a}{ }_{b c d}$. 
To derive an expression for the deformation of $\mathcal{R}^{a} b c d$, we exploit the Palatini identity, and Eq.(3.8), to write the tensor valued infinitesimal deformation of the worldsheet Christoffel symbol,

$$
\begin{aligned}
D_{\delta} \gamma_{a b}{ }^{c} & =\frac{1}{2} \gamma^{c d}\left[\nabla_{b}\left(D_{\delta} \gamma_{a d}\right)+\nabla_{a}\left(D_{\delta} \gamma_{b d}\right)-\nabla_{d}\left(D_{\delta} \gamma_{a b}\right)\right] \\
& =\gamma^{c d}\left[\nabla_{b}\left(K_{a d}{ }^{i} \Phi_{i}\right)+\nabla_{a}\left(K_{b d}{ }^{i} \Phi_{i}\right)-\nabla_{d}\left(K_{a b}{ }^{i} \Phi_{i}\right)\right] .
\end{aligned}
$$

The infinitesimal deformation in the worldsheet Riemann tensor then can be simply expressed in terms of worldsheet covariant derivatives of the $D_{\delta} \gamma_{a b}{ }^{c}$,

$$
D_{\delta} \mathcal{R}^{a}{ }_{b c d}=\nabla_{c}\left(D_{\delta} \gamma_{b d}{ }^{a}\right)-\nabla_{d}\left(D_{\delta} \gamma_{b c}{ }^{a}\right)
$$

We see that it depends on second and first worldsheet derivatives of the scalar fields $\Phi^{i}$.

The corresponding infinitesimal variations in the Ricci tensor and the scalar curvature are, respectively,

$$
\begin{gathered}
D_{\delta} \mathcal{R}_{a b}=\nabla_{c}\left(D_{\delta} \gamma_{a b}{ }^{c}\right)-\nabla_{b}\left(D_{\delta} \gamma_{a c}{ }^{c}\right) \\
D_{\delta} \mathcal{R}=\nabla^{a}\left(\gamma^{b c} D_{\delta} \gamma_{b c}{ }^{a}\right)-\nabla^{b}\left(D_{\delta} \gamma_{a b}{ }^{a}\right)-2 \mathcal{R}_{a b} K^{a b}{ }_{i} \Phi^{i} .
\end{gathered}
$$

Thus, modulo a divergence,

$$
D_{\delta} \mathcal{R}=-2 \mathcal{R}_{a b} K^{a b}{ }_{i} \Phi^{i}
$$

We also note that,

$$
D_{\delta}(\sqrt{-\gamma} \mathcal{R})=-2 \mathcal{G}_{a b} K^{a b}{ }_{i} \Phi^{i}
$$

where $\mathcal{G}_{a b}$ is the worldsheet Einstein tensor.

This concludes the analysis of the deformation of the intrinsic geometry of the worldsheet.

\section{DEFORMATIONS OF THE EXTRINSIC GEOMETRY}

The extrinsic geometry is characterized by the extrinsic curvatures, $K_{a b}{ }^{i}$, and the

extrinsic twist, $\omega_{a}^{i j}$. As a preliminary step, let us examine the gradient along the defor- 
mation vector field of the normal basis, $D_{\delta} n^{i}$, in the same way as we did for the tangent basis. We expand,

$$
D_{\delta} n_{i}=-J_{a i} e^{a}+\gamma_{i j} n^{j}
$$

This equation is the analogue for infinitesimal deformations of the Weingarten equation (2.4b). We note that $J_{a i}$ appears in Eqs.(3.2) and (4.1) in an analogous way to that of $K_{a b}{ }^{i}$ in Eqs.(2.4a) and (2.4b).

The normal projection of $D_{\delta} n_{i}$,

$$
\gamma_{i j}=g\left(D_{\delta} n_{i}, n_{j}\right)=-\gamma_{j i}
$$

is a new structure we have not encountered already. It vanishes on a hypersurface embedding, in the same way that $\omega_{a}^{i j}$ vanishes in the corresponding Weingarten equation. In contrast to $J_{a i}$ and $\beta_{a b}$, however, there is no simple relationship between $\gamma_{i j}$ and deformations of the worldsheet analogous to Eqs.(3.6) and (3.7).

The analogy between Eq. (4.1) and the Weingarten equation suggests a role for $\gamma_{i j}$ analogous to $\omega_{a}^{i j}$. In particular, $\gamma_{i j}$, like $\omega_{a}^{i j}$, transforms as a connection under normal frame rotations

$$
\gamma \rightarrow O \gamma O^{-1}+\left(D_{\delta} O\right) O^{-1}
$$

However, by an appropriate choice of $D_{\delta} O$, it is always possible to gauge $\gamma_{i j}$ away on the worldsheet. Reflecting this fact, as we will demonstrate below, $\gamma_{i j}$ will never appear explicitly in any physical quantity, although it will show up in intermediate calculations. Nonetheless, we will insist on explicit covariance under normal frame rotations. For this purpose, we introduce a covariant deformation derivative as follows,

$$
\tilde{D}_{\delta} \Psi_{i}=D_{\delta} \Psi_{i}-\gamma_{i}{ }^{j} \Psi_{j}
$$

Eq. (4.1) can then be written in the form

$$
\tilde{D}_{\delta} n_{i}=-J_{a i} e^{a}=-\left(\tilde{\nabla}_{a} \Phi_{i}\right) e^{a}
$$




\section{IV.1 Deformations of the extrinsic curvature}

Let us now evaluate the deformation of the extrinsic curvatures, $\tilde{D}_{\delta} K_{a b}{ }^{i}$. Using its definition we have that,

$$
\tilde{D}_{\delta} K_{a b}^{i}=-g\left(\tilde{D}_{\delta} n^{i}, D_{a} e_{b}\right)-g\left(n^{i}, D_{\delta} D_{a} e_{b}\right)
$$

Using Eq. (4.5), and the Gauss equation (2.5a), the first term on the right hand side is given by

$$
-g\left(\tilde{D}_{\delta} n^{i}, D_{a} e_{b}\right)=\gamma_{a b}{ }^{c} J_{c}^{i}
$$

The second term on the right hand side can be developed using the Ricci identity, as,

$$
\begin{aligned}
-g\left(n^{i}, D_{\delta} D_{a} e_{b}\right) & =-g\left(n^{i}, R\left(\delta, e_{a}\right) e_{b}\right)-g\left(n^{i}, D_{a} D_{\delta} e_{b}\right) \\
& =-g\left(n^{i}, R\left(n_{j}, e_{a}\right) e_{b}\right) \Phi^{j}-D_{a} g\left(n^{i}, D_{\delta} e_{b}\right)+g\left(D_{a} n^{i}, D_{\delta} e_{b}\right) \\
& =-g\left(n^{i}, R\left(n_{j}, e_{a}\right) e_{b}\right) \Phi^{j}-D_{a} J_{b}{ }^{i}+\beta_{b c} K_{a}{ }^{c i}+\omega_{a}{ }^{i j} J_{b j} \\
& =-g\left(n^{i}, R\left(n_{j}, e_{a}\right) e_{b}\right) \Phi^{j}-\tilde{\nabla}_{a} \tilde{\nabla}_{b} \Phi^{i}+K_{b c j} K_{a}{ }^{c i} \Phi^{j},
\end{aligned}
$$

where in the last line we have used Eqs. (3.6) and (3.7).

Therefore we find

$$
\tilde{D}_{\delta} K_{a b}{ }^{i}=-\tilde{\nabla}_{a} \tilde{\nabla}_{b} \Phi^{i}+\left[g\left(R\left(e_{a}, n_{j}\right) e_{b}, n^{i}\right)+K_{a c}{ }^{i} K_{b j}^{c}\right] \Phi^{j}
$$

Note that the change of the extrinsic curvatures under an infinitesimal deformation of the worldsheet involves second derivatives of the scalar fields $\Phi^{i}$.

The left hand side of Eq. (4.6) is manifestly symmetric in the indices $a$ and $b$. The apparent integrability condition on the right hand side,

$$
2 \tilde{\nabla}_{[a} \tilde{\nabla}_{b]} \Phi^{i}=\left[g\left(R\left(e_{a}, n_{j}\right) e_{b}, n^{i}\right)+K_{a c}{ }^{i} K_{b j}^{c}-(a \leftrightarrow b)\right] \Phi^{j},
$$

is automatically satisfied as a consequence of the Ricci integrability condition $(2.12 c)$. To show this, one needs to use the cyclic Bianchi identities for the spacetime Riemann tensor, $R_{\alpha[\beta \mu \nu]}=0$, and the identity $2 \tilde{\nabla}_{[a} \tilde{\nabla}_{b]} \Phi^{i}=\Omega_{a b}{ }_{j} \Phi^{j}$. 


\section{IV.2 Deformations of the extrinsic twist potential}

We turn now to the analysis of the deformation of the estrinsic twist $\omega_{a}^{i j}$. Unfortunately, the obvious measure of the deformation, $\tilde{D}_{\delta} \omega_{a}^{i j}$, does not transform covariantly under normal frame rotations. However, by examining $\tilde{D}_{\delta} \omega_{a}^{i j}$ itself, we can identify the appropriate addition that provides a covariant measure of the deformation.

By definition, we have that

$$
\tilde{D}_{\delta} \omega_{a}^{i j}=D_{\delta} \omega_{a}^{i j}-\gamma^{i}{ }_{k} \omega_{a}{ }^{k j}-\gamma^{j}{ }_{k} \omega_{a}{ }^{i k}
$$

where

$$
D_{\delta} \omega_{a}^{i j}=D_{\delta} g\left(D_{a} n^{i}, n^{j}\right)=g\left(D_{a} n^{i}, D_{\delta} n^{j}\right)+g\left(D_{\delta} D_{a} n^{i}, n^{j}\right) .
$$

The first term on the right hand side is

$$
\begin{aligned}
g\left(D_{a} n^{i}, D_{\delta} n^{j}\right) & =K_{a b}{ }^{i} g\left(e^{b}, D_{\delta} n^{j}\right)+\omega_{a}^{i k} g\left(n_{k}, D_{\delta} n^{j}\right) \\
& =-K_{a b}{ }^{i} J^{b j}+\omega_{a}{ }^{i k} \gamma^{j}{ }_{k} \\
& =-K_{a b}{ }^{i} \tilde{\nabla}^{b} \Phi^{j}+\omega_{a}{ }^{i k} \gamma^{j}{ }_{k} .
\end{aligned}
$$

In the second term of (4.9), using the Ricci identity, we have,

$$
\begin{aligned}
g\left(D_{\delta} D_{a} n^{i}, n^{j}\right) & =g\left(R\left(\delta, e_{a}\right) n^{i}, n^{j}\right)+g\left(D_{a} D_{\delta} n^{i}, n^{j}\right) \\
& =g\left(R\left(\delta, e_{a}\right) n^{i}, n^{j}\right)+D_{a} g\left(D_{\delta} n^{i}, n^{j}\right)-g\left(D_{\delta} n^{i}, D_{a} n^{j}\right) \\
& =g\left(R\left(\delta, e_{a}\right) n^{i}, n^{j}\right)+\nabla_{a} \gamma^{i j}+K_{a b}{ }^{j} \tilde{\nabla}^{b} \Phi^{i}-\omega_{a}{ }^{j k} \gamma^{i}{ }_{k} .
\end{aligned}
$$

where we have used (4.10) in the last line.

We find then that

$$
\tilde{D}_{\delta} \omega_{a}^{i j}=-K_{a b}{ }^{i} \tilde{\nabla}^{b} \Phi^{j}+K_{a b}{ }^{j} \tilde{\nabla}^{b} \Phi^{i}+\nabla_{a} \gamma^{i j}+g\left(R\left(n_{k}, e_{a}\right) n^{j}, n^{i}\right) \Phi^{k},
$$

or

$$
\tilde{D}_{\delta} \omega_{a}{ }^{i j}-\nabla_{a} \gamma^{i j}=-K_{a b}{ }^{i} \tilde{\nabla}^{b} \Phi^{j}+K_{a b}{ }^{j} \tilde{\nabla}^{b} \Phi^{i}+g\left(R\left(n_{k}, e_{a}\right) n^{j}, n^{i}\right) \Phi^{k} .
$$


This result indicates that the left hand side is the covariant measure of the deformation of the extrinsic twist potential. In fact, the right hand side of (4.12b) is manifestly covariant, and thus so also is the left hand side. Both sides of Eq.(4.12b) are manifestly antisymmetric in the indices $i$ and $j$. Unlike for the deformation of the extrinsic curvature, Eq.(4.6), here no integrability condition need ever be invoked. We also note the identity

$$
\tilde{D}_{\delta} \omega_{a}^{i j}-\nabla_{a} \gamma^{i j}=D_{\delta} \omega_{a}^{i j}-\tilde{\nabla}_{a} \gamma^{i j}
$$

The deformation of the curvature of the extrinsic twist is then given by

$$
D_{\delta} \Omega_{a b}^{i j}=\tilde{\nabla}_{a}\left(D_{\delta} \omega_{b}^{i j}\right)-\tilde{\nabla}_{b}\left(D_{\delta} \omega_{a}^{i j}\right) .
$$

We note that Eqs.(4.6) and (4.14) are consistent with the integrability condition, Eq.(2.12c)

This concludes the description of the deformation of the extrinsic geometry of the membrane.

\section{IV.3 Deformations of worldsheet derivatives of the extrinsic curvature}

In theories involving terms quadratic in the extrinsic curvatures, one needs to evaluate

also terms like $\tilde{D}_{\delta}\left(\tilde{\nabla}_{a} K_{c d}^{i}\right)$, to obtain the linearized equations of motion. One would like to re-express terms of this form as,

$$
\tilde{\nabla}_{a}\left(\tilde{D}_{\delta} K_{c d}^{i}\right)+\text { lower order terms }
$$

and exploit the fact that we already know what $D_{\delta} K_{a b}^{i}$ is. This involves the evaluation of the commutator, $\left[\tilde{D}_{\delta}, \tilde{\nabla}_{a}\right]$. We will do this for the commutator operating on an arbitrary worldsheet/normal frame vector, $A_{b i}$ :

$$
\begin{aligned}
\tilde{D}_{\delta} \tilde{\nabla}_{a} A_{b i}= & D_{\delta}\left[D_{a} A_{b i}-\gamma_{a b}^{c} A_{c i}-\omega_{a i}{ }^{j} A_{b j}\right]-\gamma_{i}{ }^{j} \tilde{\nabla}_{a} A_{b j} \\
= & D_{a} D_{\delta} A_{b i}-D_{\delta}\left[\gamma_{a b}^{c} A_{c i}+\omega_{a i}{ }^{j} A_{b j}\right]-\gamma_{i}{ }^{j} \tilde{\nabla}_{a} A_{b j} \\
= & D_{a} D_{\delta} A_{b i}-\gamma_{a b}^{c} D_{\delta} A_{c i}-\omega_{a i}{ }^{j} D_{\delta} A_{b j} \\
& \quad+\gamma_{a b}^{c} D_{\delta} A_{c i}+\omega_{a i}{ }^{j} D_{\delta} A_{b j}-D_{\delta}\left[\gamma_{a b}^{c} A_{c i}\right]-D_{\delta}\left[\omega_{a i}{ }^{j} A_{b j}\right]-\gamma_{i}{ }^{j} \tilde{\nabla}_{a} A_{b j} \\
= & \tilde{\nabla}_{a} D_{\delta} A_{b i}-\left(D_{\delta} \gamma_{a b}^{c}\right) A_{c i}-\left(D_{\delta} \omega_{a i}{ }^{j}\right) A_{b j}-\gamma_{i}{ }^{j} \tilde{\nabla}_{a} A_{b j} .
\end{aligned}
$$

Therefore, we find, 


$$
\left[\tilde{D}_{\delta}, \tilde{\nabla}_{a}\right] A_{b i}=-\left\{\left(D_{\delta} \gamma_{a b}^{c}\right) \delta_{i}^{j}-\left[\left(D_{\delta} \omega_{a i}{ }^{j}\right)-\left(\tilde{\nabla}_{a} \gamma_{i}{ }^{j}\right)\right] \delta_{b}^{c}\right\} A_{c j} .
$$

Note that on the right hand side appears the same covariant combination appearing in Eq. (4.13).

A useful application of this equation is given by considering the deformation of the d'Alembertian $\tilde{\Delta}=\tilde{\nabla}^{a} \tilde{\nabla}_{a}$. Applying the d'Alembertian to an arbitrary $\Psi^{i}$, one finds

$$
\begin{aligned}
\tilde{D}_{\delta}\left(\tilde{\Delta} \Psi^{i}\right) & =\left(D_{\delta} \gamma^{a b}\right) \tilde{\nabla}_{a} \tilde{\nabla}_{b} \Psi^{i}+\gamma^{a b}\left[\tilde{D}_{\delta}, \tilde{\nabla}_{a}\right] \tilde{\nabla}_{b} \Psi^{i}+\gamma^{a b} \tilde{\nabla}_{a}\left\{\left[\tilde{D}_{\delta}, \tilde{\nabla}_{b}\right] \Psi^{i}\right\}+\tilde{\Delta}\left(\tilde{D}_{\delta} \Psi^{i}\right) \\
& =\tilde{\Delta}\left(\tilde{D}_{\delta} \Psi^{i}\right)-2 \tilde{\nabla}_{a}\left[K^{a b}{ }_{j} \Phi^{j}\left(\tilde{\nabla}_{b} \Psi^{i}\right)\right]+\left[\nabla^{a}\left(K_{j} \Phi^{j}\right)\right]\left(\tilde{\nabla}_{a} \Psi^{i}\right) \\
& +2 K^{a b[i}\left(\tilde{\nabla}_{a} \Phi^{k]}\right) \tilde{\nabla}_{b} \Psi_{k}+2 \tilde{\nabla}_{a}\left[K^{a b[i}\left(\tilde{\nabla}_{b} \Phi^{k]}\right) \Psi_{k}\right] \\
& -g\left(R\left(n_{j}, e^{b}\right) n^{k}, n^{i}\right) \Phi^{j}\left(\tilde{\nabla}_{b} \Psi_{k}\right)-\tilde{\nabla}_{b}\left[g\left(R\left(n_{j}, e^{b}\right) n^{k}, n^{i}\right) \Phi^{j} \Psi_{k}\right]
\end{aligned}
$$

This expression will be useful in the following section.

\section{DYNAMICS: SOME EXAMPLES}

In this section, we apply the kinematical framework we have developed to the derivation of the equations of motion, and of the linearized equations of motion, for two phenomenological theories of relativistic membranes of physical interest. We begin with the familiar Nambu action. This will allow us to recover the results of Refs.[3] and [4]. A second example we consider is a correction term quadratic in the extrinsic curvatures.

The Nambu action for a relativistic membrane is given by,

$$
S_{0}=-\sigma \int d^{D} \xi \sqrt{-\gamma},
$$

where $\sigma$ is the membrane tension.

To derive the equations of motion, we can describe the deformations of the worldsheet with the vector field $\delta=\Phi^{i} n_{i}$, because only motions transverse to the worldsheet are physical. We have that

$$
\delta S_{0}=-\sigma \int d^{D} \xi \sqrt{-\gamma} \gamma^{a b} K_{a b}^{i} \Phi_{i}=0 .
$$

Therefore the equations of motion describing an extremal surface are given by, 


$$
K^{i}=0,
$$

and we recover the well known result that extremal surfaces have vanishing trace of the extrinsic curvatures.

To obtain the linearized equations of motion, consider

$$
\begin{aligned}
\tilde{D}_{\delta} K^{i} & =\gamma^{a b} \tilde{D}_{\delta} K_{a b}{ }^{i}+K_{a b}{ }^{i} D_{\delta} \gamma^{a b} \\
& =-\tilde{\Delta} \Phi^{i}+\left[g\left(R\left(e_{a}, n_{j}\right) e^{a}, n^{i}\right)+K_{a b}{ }^{i} K^{a b}{ }_{j}\right] \Phi^{j},
\end{aligned}
$$

so that we find the linearized equations of motion in the form,

$$
\tilde{\Delta} \Phi^{i}+\left[K_{a b}{ }^{i} K^{a b}{ }_{j}-g\left(R\left(e_{a}, n_{j}\right) e^{a}, n^{i}\right)\right] \Phi^{j}=0,
$$

in agreement with Eq.(4.1) of the second paper in Ref.[3]. This set of coupled linear equations can be seen as the equations of motion for a multiplet of scalar fields, with a "variable mass" that depends on a particular projection of the curvature of spacetime, and on the extrinsic geometry.

When the projection of the spacetime Riemann tensor vanishes, Eq. (5.3) can be written in the form, which will be used below,

$$
\mathcal{O}^{i}{ }_{j} \Phi^{j} \equiv\left[\tilde{\Delta}_{j}^{i}+K_{a b}{ }^{i} K^{a b}{ }_{j}\right] \Phi^{j}=0
$$

We now consider a less simple example involving an action quadratic in the extrinsic curvature,

$$
S_{2}=\alpha \int d^{D} \xi \sqrt{-\gamma} K_{i} K^{i}
$$

where $\alpha$ is a coefficient characterizing the rigidity of the membrane. This action is of some interest in that, modulo the totally contracted Gauss-Codazzi equation, when $D=2$, and the background geometry is flat, this action represents the most general action of this order in the worldsheet geometry. For an alternative derivation of the equations of motion corresponding to higher order actions of this order, see Ref.[9].

The variation of this action with respect to normal deformations of the worldsheet gives,

$$
\delta S_{2}=\alpha \int d^{D} \xi \sqrt{-\gamma}\left[K_{i} K^{i} K^{j} \Phi_{j}+2 K_{i}\left(-\tilde{\Delta} \Phi^{i}+g\left(R\left(e_{a}, n_{j}\right) e^{a}, n^{i}\right) \Phi^{j}-K_{a b}^{i} K_{a b j} \Phi^{j}\right)\right]
$$


Thus, the Euler-lagrange equations for $S_{2}$ are given by,

$$
\tilde{\Delta} K^{i}+\left[-g\left(R\left(e_{a}, n^{j}\right) e^{a}, n^{i}\right)+\left(\gamma^{a c} \gamma^{b d}-\frac{1}{2} \gamma^{a b} \gamma^{c d}\right) K_{a b}{ }^{j} K_{c d}{ }^{i}\right] K_{j}=0 .
$$

Note that extremal surfaces are obvious non-trivial solutions of these equations.

The linearized equations of motion are considerably more complicated than in the case of an extremal surface. For the sake of simplicity, we restrict ourselves to the case in which the background spacetime is Minkowski, in order to disregard the spacetime curvature projections. The generalization to an arbitrary background is straightforward. For this case, a lengthy computation, exploiting (4.16), gives the linearized equations of motion in the form

$$
\begin{aligned}
& -\tilde{\Delta} \tilde{\Delta} \Phi^{i}-2 K_{j}^{a b} K^{j}\left(\tilde{\nabla}_{a} \tilde{\nabla}_{b} \Phi^{i}\right)+\frac{1}{2} K^{j} K_{j} \tilde{\Delta} \Phi^{i} \\
& +\left(K^{i} K_{j}-2 K_{a b}{ }^{i} K^{a b}{ }_{j}\right) \tilde{\Delta} \Phi^{j}-2 K^{a b}{ }_{j}\left(\tilde{\nabla}_{a} K^{j}\right)\left(\tilde{\nabla}_{b} \Phi^{i}\right)-K_{j}\left(\tilde{\nabla}^{b} K^{j}\right)\left(\tilde{\nabla}_{b} \Phi^{i}\right) \\
& -2 \tilde{\nabla}^{c}\left[K_{a b}{ }^{i} K^{a b}{ }_{j}\right]\left(\tilde{\nabla}_{c} \Phi^{j}\right)+2 K^{a b i}\left(\tilde{\nabla}_{a} K_{j}\right)\left(\tilde{\nabla}_{b} \Phi^{j}\right)-2 K^{a b}{ }_{j}\left(\tilde{\nabla}_{a} K^{i}\right)\left(\tilde{\nabla}_{b} \Phi^{j}\right) \\
& +2 K_{j}\left(\tilde{\nabla}^{b} K^{i}\right)\left(\tilde{\nabla}_{b} \Phi^{j}\right)-\tilde{\Delta}\left[K_{a b}{ }^{i} K^{a b}{ }_{j}\right] \Phi^{j}-\left(\tilde{\nabla}^{a} K^{i}\right)\left(\tilde{\nabla}_{a} K_{j}\right) \Phi^{j} \\
& -2 K^{a b}{ }_{j}\left(\tilde{\nabla}_{a} \tilde{\nabla}_{b} K^{i}\right) \Phi^{j}+2 K_{a b}{ }^{i} K^{b c}{ }_{k} K^{a}{ }_{c j} K^{j} \Phi^{k}+\frac{1}{2} K_{a b}{ }^{i} K^{a b}{ }_{k} K^{j} K_{j} \Phi^{k} \\
& +K^{i} K_{j} K_{a b}^{j} K_{k}^{a b} \Phi^{k}-K_{a b}{ }^{i} K^{a b j} K_{c d j} K^{c d}{ }_{k} \Phi^{k}=0 .
\end{aligned}
$$

The scalar fields $\Phi^{i}$ satisfy then a set of coupled fourth order linear differential equations. It is interesting to note that, for a hypersurface, the square of the worldsheet d'Alembertian is the only term that depends only on the intrinsic geometry of the worldsheet.

The linearized equations (5.7) are rather complicated. An interesting special case is given by considering linearization about an extremal surface, i.e. setting $K^{i}=0$. The equations simplify considerably, and reduce to

$$
-\left(\mathcal{O}^{2}\right)^{i}{ }_{j} \Phi^{j}=0
$$

where the operator $\mathcal{O}$, defined in Eq. (5.4), is the operator describing small perturbations about an extremal surface induced by the Nambu action. It is remarkable that its square appears here. Thus, linear perturbations about an extremal surface which satisfy Eq. (5.4), continue to be solutions when one takes into account the modifications induced by the action (5.5). 
We conclude this section with the following remarks about the deformation connection $\gamma^{i j}$. We note that the action must be a scalar under normal frame rotations. Such an action (ignoring possible contractions of worldsheet or spacetime indices) involves an integrand consisting of a totally contracted product of normal frame tensors. The most simple such product is of the form $P^{i} Q_{i}$, where $P_{i}$ and $Q_{i}$ are normal frame vectors. We note that

$$
\begin{aligned}
D_{\delta}\left(P^{i} Q_{i}\right) & =P^{i} D_{\delta} Q_{i}+Q^{i} D_{\delta} P_{i} \\
& =P^{i} D_{\delta} Q_{i}+Q^{i} D_{\delta} P_{i}-\left(\gamma_{i j}+\gamma_{j i}\right) P^{i} Q^{j} \\
& =P^{i} \tilde{D}_{\delta} Q_{i}+Q^{i} \tilde{D}_{\delta} P_{i}=\tilde{D}_{\delta}\left(P^{i} Q_{i}\right),
\end{aligned}
$$

where the second line follows from the first line because of the anti-symmetry of $\gamma_{i j}$. The introduction of the normal frame covariant variation does not complicate the derivation of the Euler-Lagrange equations. Let us now denote these equations by,

$$
\mathcal{E}_{i}=0
$$

The perturbed equations of motion are then just

$$
\tilde{D}_{\delta} \mathcal{E}_{i}=0 .
$$

Modulo the background equations of motion, these equations reduce to $D_{\delta} \mathcal{E}_{i}=0$. In other words, the connection $\gamma_{i j}$ never needs to be calculated explicitly. In perturbation theory, the normal frame covariant derivative comes for free. In light of the above remarks, one can safely always set $\gamma_{i j}=0$.

\section{DISCUSSION}

In this paper we have presented a thorough analysis of the kinematics of infinitesimal deformations of the worldsheet spanned by a membrane of arbitrary dimension in any spacetime. The physical measure of the deformation is given by the normal components of the displacement vector. These normal components are scalar fields living on the worldsheet. The deformation of the intrinsic geometry is straightforward. The deformation of the extrinsic geometry, however, is complicated by the requirement of covariance under normal frame rotations. We introduce a manifestly covariant deformation operator. When we do this the covariant deformations of both the extrinsic curvature and the extrinsic 
twist curvature are given by second order hyperbolic partial differential operators acting on the scalar fields.

This kinematical framework is applied in sec. $\mathrm{V}$ to derive the equations of motion and their linearizations both for a system described by the Nambu action and for a system involving an action quadratic in the extrinsic curvature. Specializing to Minkowski spacetime for simplicity, we find that the perturbations about an extremal surface are described by a second order hyperbolic operator for the Nambu dynamics, and by its square for the dynamics described by an action quadratic in the extrinsic curvature.

A more systematic treatment of all low order actions will be addressed in a forthcoming paper [16]. We also leave for a future publication a non-perturbative description of the deformations of a relativistic membrane. This involves a non-trivial generalization of the Raychaudhuri equations for a curve [17].

\section{ACKNOWLEDGEMENTS}

We gratefully acknowledge support from CONACyT under the grant 3354-E9308.

\section{REFERENCES}

1. A. Vilenkin, Phys. Rep. 121, 263 (1985).

2. J. Garriga and A. Vilenkin, Phys. Rev. D44, 1007 (1991); ibid. D45, 3469 (1992); ibid. D47, 3265 (1993).

3. J. Guven, Phys Rev D48 4606 (1993); ibid. D48 5562 (1993); in Proceedings of SILARG VIII, ed. by P. Letelier and W. Rodriguez, (World Scientific, Singapore, 1994).

4. A.L. Larsen and V.P. Frolov, Nucl Phys B414, 129 (1994).

5. B. Carter, Phys. Rev. D48, 4835 (1993), approaches the problem from a more mathematically sophisticated point of view.

6. For example, see H. B. Lawson Lectures on Minimal Submanifolds 2nd. edition, (Pub- 
lish or Perish, 1980).

7. For example, see A. Polyakov, Nucl. Phys. B268, 406 (1986); T.L. Curtright, G.I. Ghandour, and C.K. Zachos, Phys. Rev. D34, 3811 (1986); K. Maeda and N. Turok, Phys Lett B202 376 (1988); R. Gregory, D. Haws, and D. Garfinkle, Phys. Rev. D42 343 (1990); R. Gregory, ibid. D43 520 (1991); B. Carter and R. Gregory, (Preprint, 1994).

8. P.S. Letelier, Phys. Rev. D41 1333 (1990); B. Boisseau and P.S. Letelier, ibid. D46 1721 (1992); See also C. Barrabes, B. Boisseau, and M. Sakellariadou, ibid. D49 2734 (1994).

9. B. Carter, Class. Quantum Grav. 11, 2677 (1994).

10. D.H. Hartley and R.W. Tucker, in Geometry of Low-Dimensional Manifolds: 1, London Mathematical Society Lecture Note Series 150, ed. by S.K. Donaldson and C.B. Thomas (Cambridge University Press, Cambridge, 1990).

11. L. P. Eisenhart Riemannian Geometry (Princeton Univ. Press, Princeton, 1947); M. Spivak Introduction to Differential Geometry: Vols. I to 5 (Publish or Perish, Boston MA 1970); S. Kobayashi and K. Nomizu Foundations of Differential Geometry: Volume II (Interscience, New York 1969).

12. M. Dajczer, Submanifolds and Isometric Immersions (Publish or Perish, Houston, Texas, 1990).

13. B. Carter, Journal of Geometry and Physics 8, 52 (1992).

14. B. Carter, Class. Quantum Grav. 9, S19 (1992).

15. See, for example, S.W. Hawking and G.F.R. Ellis The Large Scale Structure of SpaceTime (Cambridge Univ. Press, Cambridge, 1973).

16. R. Capovilla and J. Guven, (to appear).

17. R. Capovilla and J. Guven, (gr-qc/9411061). Phys. Rev. D (To appear 1995). 